\title{
REVIEW
}

\section{Association between the Functional Independence Measure following spinal cord injury and long-term outcomes}

\author{
JT Cohen ${ }^{1}$, RJ Marino ${ }^{2}$, P Sacco $^{3}$ and N Terrin ${ }^{1}$
}

\begin{abstract}
Study design: Retrospective cohort.
Objectives: To estimate the association between the Functional Independence Measure (FIM) for spinal cord injury (SCI) patients at time of discharge from rehabilitation and long-term resource utilization, residential status and employment. The intention was to assess the value of FIM for projecting economic burden in SCl.

Setting: $\quad$ Federally designated spinal cord injury model system facilities throughout the USA.

Methods: We analyzed data from the National Spinal Cord Injury Statistical Center database $(n=14620)(1988-2010)$, excluding subjects with: age $<6$ years, normal motor function, death before discharge or etiology from gunshot or penetrating wound ( $n=11685$ retained). We investigated the association between motor FIM at rehabilitation discharge and residential status, survival and outcomes at 1, 5, 10, 15 and 20 years follow-up, including FIM, residential status, hospitalizations, days hospitalized in previous year, daily paid and total care and paid hours worked. Regression controlled for injury completeness, neurological level, demographic characteristics and temporal effects.

Results: $\quad$ All outcomes were statistically associated with higher FIM scores at discharge. Each one-point increment in FIM was associated with improvements in: probability of institution care at discharge $(-0.34 \%)$ and at follow-up $(-0.13 \%)$, FIM score at follow-up (0.76 points), hospitalizations and days hospitalized/year ( -0.0044 and -0.071 , respectively), probability of needing paid assistance $(-0.72 \%)$ or any assistance $(-0.85 \%)$ and probability of paid work $(0.41 \%)$.
\end{abstract}

Conclusion: The FIM at discharge has predictive value for long-term outcomes. Improvement in FIM suggests reduced economic burden in $\mathrm{SCl}$ patients.

Sponsorship: Novartis Pharmaceuticals Corporation.

Spinal Cord (2012) 50, 728-733; doi:10.1038/sc.2012.50; published online 29 May 2012

Keywords: spinal cord injury; Functional Independence Measure; cost; economic burden; hospitalization, employment.

\section{INTRODUCTION}

Each year in the United States, an estimated 11000 individuals experience traumatic spinal cord injury (SCI). ${ }^{1}$ These individuals will incur short- and long-term expenses for medical treatment and rehabilitation. Emergency treatment reduces the damage caused by SCI (immobilization of the spine, maintenance of body functions and prevention of infection), and surgery can improve function in some situations (for example, to address compression of the spinal cord by a herniated disk). Pharmacological therapies aimed at improving long-term function, however, are limited. For example, results from two randomized trials lead the American Academy of Neurological Surgeons to conclude that nerve growth agent GM-1 monosialoganglioside (for example, Sygen, produced by Fidia Pharmaceuticals, Abano Terme, Padova, Italy) fails to confer a clinical benefit. $^{2}$ Conclusions regarding methylprednisolone (Solu-Medrol, produced by Pfizer Pharmaceuticals, New York, NY, USA) have been less definitive, but here too the American Academy of Neurological Surgeons concluded that the evidence does not support a clinical benefit. ${ }^{2}$ Individuals who sustain a SCI are at risk for secondary complications resulting in increased use of health care resources. Additionally, persons with substantial disability may require assistance with activities of daily living and have a reduced capacity for paid work. Therefore, a major consequence of disability because of SCI is increased cost to the patient, the health care system and society.

With limited options available to improve the condition of SCI patients, there has been little impetus to systematically and quantitatively estimate the benefits of potential SCI therapies. As a practical matter, it is likely that the long-term benefits of novel therapies would have to be estimated by projecting gains as a function of short-term outcomes measured in clinical trials. In this paper we estimate the association between the motor domain of the Functional Independence Measure (FIM) at the time of discharge from rehabilitation and longer-term quality of life and care costs. Although the target of novel therapies is likely to be neurological function, we chose the FIM as a more functionally relevant measure. Understanding the association between short and long-term outcomes will begin to aid estimation of the economic impact of improved functional ability in persons with a traumatic SCI.

\section{MATERIALS AND METHODS}

Data source

The National Spinal Cord Injury Statistical Center (NSCISC) database at the University of Alabama, one of the most extensive registries of SCI patients, contains data on patients treated since 1973 at federally designated model SCI

${ }^{1}$ Institute for Clinical Research and Health Policy Studies, Tufts Medical Center, Boston, MA, USA; ${ }^{2}$ Thomas Jefferson Hospital, Philadelphia, PA, USA and ${ }^{3}$ Novartis Pharmaceuticals Corporation, East Hanover, NJ, USA

Correspondence: Dr JT Cohen, Institute for Clinical Research and Health Policy Studies, Tufts Medical Center, 800 Washington Street, \#063, Boston, MA 02111, USA Email: jcohen@TuftsMedicalCenter.org

Received 23 December 2011; revised 24 February 2012; accepted 27 March 2012; published online 29 May 2012 
care systems throughout the United States. ${ }^{3}$ A total of 26 care centers have contributed to the NSCISC since its inception. Funding for continued participation is subject to renewal every 5 years, so the composition of the contributing centers shifts. At this time, there are 14 centers contributing to the NSCISC, centers that together treat $\sim 15 \%$ of all new cases in the United States of SCI. ${ }^{4}$

Using data through March 2010 for patients admitted no earlier than 1 October 1988 (when FIM scores were first recorded), we excluded subjects for whom (1) age was 5 years or less at time of injury (FIM score is not recorded below the age of 6 years), (2) injury impairment was classified as E using the American Spinal Injury Association (ASIA) scale ${ }^{5}$ or the corresponding level as measured using the Frankel scale (that is, we excluded subjects with normal motor function), (3) death occurred before discharge from rehabilitation or (4) etiology was gunshot wound or penetrating wound. We excluded gunshot and penetrating wound etiologies, because subjects with these injuries are often excluded from SCI pharmaceutical clinical trials (for example, see Bracken et $a .^{6}$ and Geisler et al. ${ }^{7}$ ). Retaining this population would therefore make projections developed here less applicable to results from these and other clinical trials.

\section{The FIM score}

The FIM is a popular measure in the United States for assessment of SCI patients, because it is required in order to obtain reimbursement from Medicare. ${ }^{8}$ The FIM's properties have been extensively documented, ${ }^{9-13}$ and its ability to detect meaningful changes in function during rehabilitation has been verified. ${ }^{14,15}$ The motor domain of the FIM score is the sum of the ratings for each of 13 tasks. ${ }^{16}$ Each task rating ranges from one (full assistance required) to seven (complete independence). Hence, the total motor domain FIM score (hereafter referred to as the FIM) ranges from 13 (least favorable) to 91 (most favorable). As there is a high correlation among the 13 FIM motor tasks, ${ }^{16}$ this analysis uses the sum of these scores. To adjust for a systematic decrease in FIM scores following adoption of a prospective payment system by inpatient rehabilitation facilities in January, 2002, we decreased pre-2002 total FIM scores by $2.77 .{ }^{17}$

\section{Analysis}

Outcomes. The analysis investigated the association between FIM measured at the time of release from rehabilitation and both one-time outcomes and outcomes measured at repeated follow-up times. One-time outcomes included: (1) residential status at discharge from rehabilitation, categorized as 'community' (reported in the NSCISC database as private residence, group living situation or hotel/motel) or 'professional care' (reported in the NSCISC database as hospital or nursing home) and (2) survival. Follow-up outcomes reported potentially at $1,5,10,15$ and 20 years post injury included: (3) FIM score, (4) residential status (again, dichotomized as 'community' or 'professional care'), (5) number of hospitalizations during the previous year, (6) number of days hospitalized during the previous year, (7) hours of paid care assistance received per day, (8) total hours of care assistance received per day (paid and informal unpaid) and (9) paid work hours per week. As explained in the Technical Appendix (in Supplementary Information), we dichotomized the last three of these outcomes for statistical reasons $(0$ or $>0)$.

Outcome-specific inclusion criteria. For paid hours of care assistance per day (outcome 7), total hours of assistance per day (outcome 8), and paid hours worked per week (outcome 9), we restricted the analysis to observations for which residential status was classified as 'community', because these outcomes have either no meaning or a noncomparable meaning among subjects who are institutionalized.

Statistics. We regressed outcomes against the FIM using statistically appropriate techniques (see Technical Appendix (in Supplementary Information) for details). Binary outcomes included residential status at discharge (outcome 1) and at follow-up (outcome 4). For statistical reasons, we also converted paid hours of assistance (outcome 7), total hours of assistance (outcome 8) and paid work hours (outcome 9) to binary variables, dichotomizing these quantities as 0 or $>0$. Negative binomial outcomes included number of hospitalizations (outcome 5) and number of days hospitalized (outcome 6). We modeled FIM at follow-up using linear regression (outcome 3). Finally, we used a Cox proportional hazards model to model survival. For follow-up outcomes potentially measured on multiple occasions for each subject (outcomes 3-9), we used a generalized linear model with a robust variance estimator.

Controlled variables. In addition to the FIM, the analysis controlled for the following characteristics. First, it controlled for both ASIA grade (injury completeness) and neurological level (that is, spinal column location where the injury occurred). In cases where the Frankel injury completeness level was reported, we replaced this information with the corresponding ASIA grade. As noted earlier, injury completeness and neurological level are correlated with outcomes of interest. ${ }^{18-20}$ As the goal of this study is to estimate the incremental implications of changes in the FIM score (independent of changes in injury completeness and neurological level) in terms of longerterm outcomes, our analysis controls for these characteristics.

In order to capture possible interactions between injury completeness and neurological level while creating a reasonable number of joint categories, we used categories based largely on Sipski et al. ${ }^{21}$ For example, the first injury completeness/neurological level category includes all subjects with injuries at cervical levels $1-4$ (that is, C1-C4) and completeness grades of A (complete) or $\mathrm{B}$ (incomplete with sensory function only preserved below the point of injury).

We also controlled for demographic characteristics because these factors can influence outcomes of interest, such as survival and the number of rehospitalizations. ${ }^{18-21}$ Specifically, we controlled for gender, race, Hispanic ethnicity and marital status for all outcomes. In addition, for outcomes measured repeatedly at follow-up, we controlled for age at follow-up, while for outcomes not measured repeatedly at follow-up (survival time and residential status at discharge), we controlled for age at the time of injury. Finally, the analysis controls for year of admission to rehabilitation to address the possibility that practices change. For example, studies have reported a decline in the length of stay for rehabilitation over time. ${ }^{22}$

As there are a large number of observations in the data set and hence ample degrees of freedom, we retained all predictors in the models regardless of the statistical significance of their regression coefficients.

Imputation of missing values. As explained in greater detail in the Technical Appendix (in Supplementary Information), we imputed missing values for FIM measured at discharge and for all outcomes except for date of death. Next, we created five imputed data sets, eliminating from each the imputed followup values for time points beyond the date when the subject died or beyond the date when the data set was frozen (March 2010). We conducted each regression on each of the five imputed data sets and used appropriate techniques to combine the resulting estimates quantifying the association between FIM and each outcome.

Absolute impact of FIM score change on outcomes. This study estimated the absolute impact of a one-point change in FIM score at discharge from rehabilitation on outcomes, because health economic outcomes of interest are typically proportional to these absolute changes. For example, savings stemming from reduced institutionalization is proportional to the absolute change in the probability of institutionalization. The impact on the odds ratio cannot be used to directly inform economic analyses. Nonlinear regression outcomes for which we computed the absolute impact of a one-point FIM score change included residential status at discharge from rehabilitation, residential status at follow-up, hospitalizations per year, days hospitalized per year, any paid care assistance, any assistance and any paid work.

As explained in the Technical Appendix (in Supplementary Information), the absolute impact of a one-point FIM score change varies across subjects. To estimate a single value for the absolute impact of a one-point FIM score change, we therefore averaged the impacts estimated individually for every subject in the NSCISC database included in our analysis.

\section{Statement of ethics}

Use of the de-identified NSCISC data was approved by the Institutional Review Board at Tufts Medical Center. 


\section{RESULTS}

We retrieved data for 14620 subjects from the NSCISC database. Of these subjects, we excluded 2935, because of at least one of the following exclusion criteria: their injury impairment was classified as 'normal' (ASIA grade of 'E') $(n=214)$, death occurred before discharge $(n=20)$ or the injury was caused by a gunshot or deep penetrating wound $(n=2712)$ (note that the exclusion categories are not mutually exclusive). Tables 1 and 2 summarize the characteristics of the retained sample. As detailed in Table 2, the available number of subjects drops from 9644 at 1-year follow-up to 191 at 20 years follow-up. The number of subjects with entries in any particular field at each follow-up time point was below these limits.

Table 3 reports the impact on each outcome of a one-point FIM score change at discharge from rehabilitation and the central estimates for the regression coefficients. As detailed in the Technical Appendix (in Supplementary Information), Table A-1, the confidence intervals are narrow, extending no more than $23 \%$ above and below the central estimate value in all cases. The Technical Appendix (in Supplementary Information) also shows that for all but two outcomes, estimates of the impact of a change in the FIM score developed using the imputed data did not differ substantially from estimates developed using the original data. For hospitalizations per year and days hospitalized per year, however, the difference between the impacts computed using the original data were substantially greater than the impacts computed using the imputed data, approaching nearly a factor of two for days hospitalized per year.

Finally, Table 4 reports the impact of one-point FIM score change on follow-up outcomes by year of follow-up. Technical Appendix Table A-2 (in Supplementary Information) lists the confidence intervals for the central estimates in Table 4 .

\section{DISCUSSION}

The results from this analysis show that higher values of FIM measured at the time of discharge from rehabilitation are associated with both intermediate and long-term outcomes. In the short term, a higher FIM score is associated with a greater probability of living in the community, rather than in an institutionalized setting. Over the longer term, a higher FIM at rehabilitation discharge is associated with longer survival, improved function at follow-up (that is, higher FIM scores at follow-up), reduced care burden (lower probability of institutionalization, less hospitalization and fewer hours of both paid and informal assistance) and a greater probability of having paid work.

Heterogeneity across treatment centers and among individuals makes it inappropriate to use our findings to predict outcomes for specific individuals. Nonetheless, the findings described here can be used to project both intermediate and long-term population average benefits of therapy based on a clinical trial reporting only the much shorter term FIM score at discharge from rehabilitation. For example, a hypothetical therapy that on average achieves a fivepoint improvement in the motor domain total FIM score would reduce the proportion of individuals institutionalized at discharge by $1.7 \%$, and the proportion of individuals institutionalized at follow-up from between 0.6 to $0.8 \%$. Hospitalizations per year per individual would drop by 0.02 (that is, 2 per 100 individuals), and days hospitalized per year would on average drop by more than 0.3 . The proportion of individuals receiving paid assistance each day would decrease by $3.5 \%$, while the proportion of individuals receiving any assistance each day (paid or unpaid) would decrease by more than $4 \%$. Finally, the proportion of individuals with any paid work would increase by $2 \%$.
Table 1 Subject characteristics-characteristics measured once

\begin{tabular}{lcccc}
\hline Quantitative (continuous) characteristics & $25^{\text {th }}$ Pctl & Median & $75^{\text {th }}$ Pctl \\
\hline Age at injury & 24 & 37 & 51 \\
FIM at rehabilitation discharge a , missing & 30 & 56 & 72
\end{tabular}

$n=1007$

$\begin{array}{ll}\text { Survival rate after discharge, (Kaplan-Meyer) } & \\ 5 \text { years } & 88.7 \% \\ 10 \text { years } & 82.2 \% \\ 15 \text { years } & 76.5 \% \\ 20 \text { years } & 73.5 \%\end{array}$

Categorical Characteristics Frequency Percentage

$A S I A$ and neurological level

$\begin{array}{lll}\mathrm{C} 1-4, \mathrm{AB} & 1429 & 12.2\end{array}$

C1-4, CD

1494

12.8

C5, AB

779

6.7

$C 5, C D$

1195

10.2

C6, AB

602

C6, $C D$

554

5.2

$C 7, A B$

248

$C 7, C D$

C8-T1, AB

310

C8-T1, CD

224

T2-T8, AB

239

1352

T2-T8, CD

329

T9-T12, AB

1054

T9-T12, CD

550

LS

Unknown

1190

136

Gender

Male

9071

Female

2614

Race

Caucasian

8553

73.2

African American or Black

2201

Native American, Eskimo or Aleut

61

Asian or Pacific Islander

Other, unclassified

Unknown

282

3.0

Ethnicity

Not of Hispanic origin

Hispanic or Latino origin

10498

1044

89.8

Unknown

143

Marital status

Single

Married

5145

4444

44.0

Divorced

1303

38.0

Separated

Widowed

333

11.2

Unknown, other-unclassified

Abbreviations: ASIA, American Spinal Injury Association; C, cervical injuries; FIM, Functional Independence Measure; LS, lumbar or sacral injuries; T, thoracic injuries.

a Motor component of the Functional Independence Score at discharge. Integer values range from 13 (maximum dependence) to 91 (maximum independence)

bThe ASIA scale classifies injuries as A (complete), B (incomplete but function limited to sensation below the point of injury), $\mathrm{C}$ (incomplete with limited motor function below the point of injury), and $\mathrm{D}$ (incomplete with more extensive motor function below the point of injury. 
Table 2 Subject Characteristics: characteristics measured at follow-up

\begin{tabular}{|c|c|c|c|c|c|c|c|c|c|c|c|c|c|c|c|}
\hline \multirow{3}{*}{$\begin{array}{l}\text { Total observations } \\
\text { Continuous and count variables }\end{array}$} & \multicolumn{3}{|c|}{ Year 1} & \multicolumn{3}{|c|}{ Year 5} & \multicolumn{3}{|c|}{ Year 10} & \multicolumn{3}{|c|}{ Year 15} & \multicolumn{3}{|c|}{ Year 20} \\
\hline & \multicolumn{3}{|c|}{$N=9644$} & \multicolumn{3}{|c|}{$N=5323$} & \multicolumn{3}{|c|}{$N=2763$} & \multicolumn{3}{|c|}{$\mathrm{N}=1144$} & \multicolumn{3}{|c|}{$\mathrm{N}=191$} \\
\hline & $\mathrm{N}$ & Mean & s.d. & $\mathrm{N}$ & Mean & s.d. & $\mathrm{N}$ & Mean & s.d. & $\mathrm{N}$ & Mean & s.d. & $\mathbf{N}$ & Mean & s.d. \\
\hline FIM & 5335 & 62.0 & 25.9 & 3168 & 63.1 & 25.7 & 1719 & 63.9 & 25.3 & 810 & 65.0 & 25.0 & 132 & 66.7 & 25.4 \\
\hline Rehospitalizations & 8218 & 0.5 & 0.9 & 4129 & 0.4 & 0.9 & 2013 & 0.4 & 0.9 & 901 & 0.4 & 0.8 & 137 & 0.3 & 0.7 \\
\hline Days rehospitalized & 8069 & 5.8 & 19.7 & 4032 & 4.2 & 16.1 & 1981 & 4.4 & 16.9 & 883 & 4.1 & 17.7 & 137 & 2.5 & 7.7 \\
\hline Paid assistance, hours per day ${ }^{b}$ & 5158 & 2.6 & 5.4 & 3399 & 2.7 & 5.7 & 1873 & 2.9 & 6.0 & 857 & 2.5 & 5.6 & 135 & 1.9 & 4.8 \\
\hline Total assistance, hours per day ${ }^{b}$ & 5129 & 7.2 & 9.4 & 3387 & 5.6 & 8.4 & 1866 & 5.4 & 8.5 & 853 & 4.9 & 7.9 & 135 & 4.4 & 7.9 \\
\hline Time at a paid job, hours per week & 5187 & 5.8 & 14.4 & 3414 & 9.4 & 17.7 & 1901 & 11.9 & 19.1 & 866 & 13.7 & 20.5 & 135 & 15.1 & 21.0 \\
\hline Categorical characteristics & Total N & & Percentage & Total N & & Percentage & Total N & & Percentage & Total N & & Percentage & Total N & & Percentage \\
\hline Residential status (community) & 8305 & & 95.2 & 4172 & & 96.7 & 2026 & & 97.3 & 907 & & 98.0 & 137 & & 99.3 \\
\hline Paid assistance $>0^{b}$ & 5158 & & 32.4 & 3399 & & 34.3 & 1873 & & 34.0 & 857 & & 30.8 & 135 & & 27.4 \\
\hline Total assistance $>0^{\mathrm{b}}$ & 5129 & & 60.7 & 3387 & & 52.9 & 1866 & & 50.8 & 853 & & 47.5 & 135 & & 44.4 \\
\hline Time at a paid job $>0^{b}$ & 5187 & & 16.9 & 3414 & & 26.4 & 1901 & & 31.9 & 866 & & 35.6 & 135 & & 38.5 \\
\hline
\end{tabular}

Abbreviation: FIM, Functional Independence Measure.

These values represent the number of follow-up observations for all subjects meeting this study's primary inclusion criteria.

${ }^{b}$ Computation of hours of paid assistance, total hours of assistance, and time at job reflects observations for which the NSCISC database reported the outcome of interest and the subject resided

in the community at follow-up (that is, the NSCISC database reported residential status as private residence, group living situation or hotel/motel).

Table 3 Regression results: change in outcomes associated with one-point change in FIM score at discharge from rehabilitation

\begin{tabular}{lcc}
\hline Outcome & $\begin{array}{c}\text { Impact of one-FIM } \\
\text { point on event }{ }^{\text {a }}\end{array}$ & $\begin{array}{c}\text { Central estimate for } \\
\text { regression coefficient }\end{array}$ \\
\hline Probability of institutional & $-0.34 \%$ & -0.040 \\
care at discharge & $(1)$ & -0.016 \\
Survival & 0.76 & 0.76 \\
FIM score at follow-up & $-0.14 \%$ & -0.050 \\
Probability of institutional & & \\
care at follow-up & -0.0044 & -0.0075 \\
Hospitalizations per year & -0.071 & -0.0080 \\
Days hospitalized per year & $-0.72 \%$ & -0.048 \\
Probability of any paid care assistance & $-0.85 \%$ & -0.049 \\
Probability of any care assistance & $0.41 \%$ & 0.022 \\
Probability of any work & & \\
for pay & &
\end{tabular}

Abbreviation: FIM, Functional Independence Measure.

aFor continuous and count data outcomes other than survival (FIM score at follow-up, hospitalizations per year, days hospitalized per year), the reported value represents the change in the outcome corresponding to a one-point change in the FIM score. For dichotomous events (institutional care at discharge, institutional care at follow-up, any paid care assistance, any care assistance and any work for pay), the impact is the change in the probability of the outcome for each one-point FIM score change. As the survival function is semi-parametric, it is not possible to compute an average change in survival duration corresponding to a one-point FIM score change. Technical Appendix Table A-1 (in Supplementary Information) reports sensitivity analysis results (impacts computed with original data, rather than imputed data). bTechnical Appendix Table A-1 (in Supplementary Information) lists confidence intervals.

Health care savings resulting from these hypothetical improvements can also be estimated. Taking the average cost of a semi-private room in a nursing home facility to be $\$ 198$ per day ${ }^{23}$ implies that the $1.7 \%$ reduction in institutionalization following rehabilitation would save an average of more than $\$ 1200$ per treated individual in the first year following injury. The $0.6-0.8 \%$ institutionalization reduction during subsequent follow-up years would save from $\$ 430$ to $\$ 580$ annually per treated individual. Hospitalization stays for an individual with SCI cost on average $\$ 20000$ (the average charge is $\$ 40000$ ). ${ }^{24}$ Hence, reducing the number of hospital stays by 0.02 per year would save $\$ 400$ annually per treated individual. A home health aid costs $\$ 21$ per hour. ${ }^{23}$ Results in Table 2 indicate that all individuals with SCI receive an average of $2.6 \mathrm{~h}$ per day of paid assistance in the first year following their injury but that this help is used by $32.4 \%$ of individuals with SCI. Taken together, these results imply that conditional on receiving any paid assistance, individuals with SCI receive an average of $\sim 8 \mathrm{~h}$ per day of paid help (that is, $2.6 \mathrm{~h}$ divided by $32.4 \%$ ). Estimates for other follow-up years are generally similar. Those individuals receiving any paid home help therefore cost an average of $\sim \$ 60000$ per year. A 3.5\% reduction in paid home health aid use would therefore save $\sim \$ 2200$ annually per treated individual.

These findings are subject to certain limitations. First, the multiple imputation algorithm that was most appropriate for this analysis treated all variables as normally distributed. In the case of dichotomous outcomes, imputed values had to be rounded before the regression analysis was conducted (values $<0.5$ were converted to zero, whereas other values were converted to one). Although this issue does introduce some bias, Bernaards et al. ${ }^{25}$ showed that omitting incomplete observations can introduce even greater bias. Importantly, the results we computed using the imputed data were in most cases very similar to the results we computed using the original data. The most extreme difference arose in the analysis of days hospitalized per year at follow-up. Even in this case, the result computed using the imputed data and the result computed using the original data were within a factor of $<2$. The results based on the imputed data should be viewed as more valid as patients lost to follow-up may differ systematically from those who are followed.

Second, adjusting for injury completeness may have resulted in over control of confounders. Recall that a key motivation for this analysis is projecting long-term benefits associated with short-term improvements in the FIM so that the FIM can be used to more comprehensively evaluate the benefits of SCI therapies. If novel therapies can influence injury completeness, then controlling for injury completeness, as done here, would obscure benefits estimated using the FIM and the relationships developed in this paper. The approach used here can hence be regarded as being conservative, because it potentially understates the magnitude of the long-term benefits associated with FIM score improvements. 
Table 4 Change at follow-up associated with a one-point change in FIM score at discharge from rehabilitation

Follow-up year

\begin{tabular}{|c|c|c|c|c|c|}
\hline & \\
\hline & 1 & 5 & 10 & 15 & 20 \\
\hline FIM at follow-up & 0.77 & 0.75 & 0.76 & 0.76 & 0.77 \\
\hline Probability of institutional care & $-0.15 \%$ & $-0.14 \%$ & $-0.13 \%$ & $-0.12 \%$ & $-0.12 \%$ \\
\hline Hospitalizations per year & -0.0045 & -0.0044 & -0.0043 & -0.0042 & -0.0042 \\
\hline Days hospitalized per year & -0.070 & -0.070 & -0.071 & -0.072 & -0.074 \\
\hline Probability of any paid care assistance & $-0.73 \%$ & $-0.73 \%$ & $-0.72 \%$ & $-0.71 \%$ & $-0.71 \%$ \\
\hline Probability of any care assistance & $-0.85 \%$ & $-0.86 \%$ & $-0.86 \%$ & $-0.86 \%$ & $-0.85 \%$ \\
\hline Probability of any work for pay & $0.39 \%$ & $0.41 \%$ & $0.43 \%$ & $0.45 \%$ & $0.47 \%$ \\
\hline
\end{tabular}

Abbreviation: FIM, Functional Independence Measure.

Technical Appendix Table A-2 (in Supplementary Information) lists confidence intervals.

Third, the estimated absolute impact of FIM score on outcomes was calculated based on the assumption that the NSCISC database is representative of the United States population of individuals with SCI. Go et al. ${ }^{26}$ found the NSCISC database to be generally similar to the population with SCI as documented in state population registries, although more severe injuries, non-Caucasians, and injuries resulting from acts of violence were very modestly over-represented in the NSCISC database. Changes in the participating care centers over time has also limited follow-up in some cases, a factor we attempted to address by use of multiple imputation techniques, as discussed above. In any case, the NSCISC database is the most extensive data set in the United States for the SCI population.

Finally, this analysis excluded individuals with SCI resulting from gunshot wounds or deep penetrating injuries, because this group is often excluded from therapeutic clinical trials for SCI treatments. As a result, our findings cannot be generalized to this group.

Despite these limitations, this work provides a useful starting point for using FIM data collected in a relatively short-term clinical trial to project longer-term outcomes. In the future, it may be possible to improve upon the work described here by using an instrument better designed to evaluate function in people with SCI. For example, the Spinal Cord Independence Measure is more sensitive to functional changes in this population than the $\mathrm{FIM},{ }^{27,28}$ but has yet to be recorded over an extended time period in a large population. We therefore encourage incorporation of the Spinal Cord Independence Measure into data collection efforts designed to track large numbers of individuals with SCI over an extended time period. In the meantime, the relationships reported here provide a useful projection of long-term outcomes so that decision makers can better understand the overall value of therapies for the SCI population.

\section{CONFLICT OF INTEREST}

Drs Cohen and Terrin received grant support from Novartis Pharmaceutical Corporation. They retained control over the methodology of the study and the decision to proceed with publication. Dr Marino serves as a consultant to Novartis Pharma AG by and through the Department of Rehabilitation Medicine at Thomas Jefferson University, Philadelphia PA. Ms Sacco is employed by Novartis Pharmaceutical Corporation.

\section{ACKNOWLEDGEMENTS}

This study was sponsored by Novartis Pharmaceuticals Corporation. The authors also acknowledge provision of data and technical assistance by the National Spinal Cord Injury Statistical Center (NSCISC) at the University of Alabama, the model systems that provide data to the NSCISC database, and support of the NSCISC database by the United States Department of Education (DOE), Office of Special Education and Rehabilitative Services (OSERS) and the National Institute on Disability and Rehabilitation Research (NIDRR).

1 Kirshblum S. Rehabilitation of spinal cord injury. In: DeLisa J, Gans B, Walsh N, Bockenek W, Pease W and Frontera W et al. (eds). Physical Medicine and Rehabilitation: Principles and Practice, 4th edn, Vol. 2. Lippincott Williams \& Wilkins: Philadelphia, PA, 2005, pp 1715-1752.

2 Anonymous. Guidelines for management of acute cervical spinal injuries. Introduction. Neurosurgery 2002; 50(3 Suppl): S1.

3 Anonymous. Annual Report for the Model Spinal Cord Injury Care Systems. National Spinal Cord Injury Statistical Center: Birmingham, AL, 2007.

4 Cao Y, Chen Y, DeVivo M. Lifetime direct costs after spinal cord injury. Top Spinal Cord Inj Rehabil 2011; 16: 10-16.

5 Standard Neurological Classification of Spinal Cord Injury. American Spinal Injury Association 2006 March 1 (cited 8 February 2010); Available from: URL: http://www.asia-spinalinjury.org/publications/2006_Classif_worksheet.pdf.

6 Bracken MB, Shepard MJ, Collins WF, Holford TR, Young W, Baskin D et al. A randomized, controlled trial of methylprednisolone or naloxone in the treatment of acute spinal-cord injury. Results of the Second National Acute Spinal Cord Injury Study. N Engl J Med 1990; 322: 1405-1411.

7 Geisler FH, Coleman WP, Grieco G, Poonian D. The Sygen multicenter acute spinal cord injury study. Spine 2001; 26(24 Suppl): S87-S98.

8 Anderson K, Aito S, Atkins M, Biering-Sorensen F, Charlifue S, Curt A et al. Functional recovery measures for spinal cord injury: an evidence-based review for clinical practice and research. J Spinal Cord Med 2008; 31: 133-144.

9 Dodds TA, Martin DP, Stolov WC, Deyo RA. A validation of the functional independence measurement and its performance among rehabilitation inpatients. Arch Phys Med Rehabil 1993; 74: 531-536.

10 Granger CV, Hamilton BB, Linacre JM, Heinemann AW, Wright BD. Performance profiles of the functional independence measure. Am J Phys Med Rehabil 1993; 72: 84-89.

11 Heinemann AW, Linacre JM, Wright BD, Hamilton BB, Granger C. Relationships between impairment and physical disability as measured by the functional independence measure. Arch Phys Med Rehabil 1993; 74: 566-573.

12 Linacre JM, Heinemann AW, Wright BD, Granger CV, Hamilton BB. The structure and stability of the Functional Independence Measure. Arch Phys Med Rehabil 1994; 75: 127-132

13 Heinemann AW, Kirk P, Hastie BA, Semik P, Hamilton BB, Linacre JM et al. Relationships between disability measures and nursing effort during medical rehabilitation for patients with traumatic brain and spinal cord injury. Arch Phys Med Rehabil 1997; 78: 143-149.

14 Granger CV, Cotter AC, Hamilton BB, Fiedler RC, Hens MM. Functional assessment scales: a study of persons with multiple sclerosis. Arch Phys Med Rehabil 1990; 71: 870-875.

15 Heinemann AW, Linacre JM, Wright BD, Hamilton BB, Granger C. Prediction of rehabilitation outcomes with disability measures. Arch Phys Med Rehabil 1994; 75 $133-143$

16 Hall KM, Cohen ME, Wright J, Call M, Werner P. Characteristics of the Functional Independence Measure in traumatic spinal cord injury. Arch Phys Med Rehabil 1999, 80: 1471-1476.

17 Qu H, Shewchuk RM, Chen Y, Deutsch A. Impact of Medicare prospective payment system on acute rehabilitation outcomes of patients with spinal cord injury. Arch Phys Med Rehabil 2011; 92: 346-351.

18 De Vivo M. Discharge disposition from model spinal cord injury care system rehabilitation programs. Arch Phys Med Rehabil 1999; 80: 785-790. 
19 DeVivo M, Krause JS, Lammertse DP. Recent trends in mortality and causes of death among persons with spinal cord injury. Arch Phys Med Rehabil 1999; 80: 1411-1419.

20 Eastwood EA, Hagglund KJ, Ragnarsson KT, Gordon WA, Marino RJ. Medical rehabilitation length of stay and outcomes for persons with traumatic spinal cord injury-1990-1997. Arch Phys Med Rehabil 1999; 80: 1457-1463.

21 Sipski ML, Jackson AB, Gomez-Marin O, Estores I, Stein A. Effects of gender on neurologic and functional recovery after spinal cord injury. Arch Phys Med Rehabil 2004; 85: 1826-1836.

22 De Vivo M. Sir Ludwig Guttmann Lecture: trends in spinal cord injury rehabilitation outcomes from model systems in the United States: 1973-2006. Spinal Cord 2007; 45: 713-721.

23 De Vivo M, Chen Y, Mennemeyer S, Deutsch A. Costs of care following spinal cord injury. Top Spinal Cord Inj Rehabil 2011; 16: 1-9.
24 De Vivo M, Farris V. Causes and costs of unplanned hospitalizations among persons with spinal cord injury. Top Spinal Cord Inj Rehabil 2011; 16: 53-61.

25 Bernaards CA, Belin TR, Schafer JL. Robustness of a multivariate normal approximation for imputation of incomplete binary data. Stat Med 2007; 26: 1368-1382.

26 Go B, DeVivo M, Richards J. The epidemiology of spinal cord injury. In: Stover S, DeLisa J and Whiteneck G (eds). Spinal Cord Injury: Clinical Outcomes from the Model Systems. Aspen Publishers: Gaithersburg, MD, 1995, pp 21-55.

27 Catz A, Itzkovich M, Agranov E, Ring H, Tamir A. SCIM-spinal cord independence measure: a new disability scale for patients with spinal cord lesions. Spinal Cord 1997; 35: 850-856.

28 Catz A, Itzkovich M, Agranov E, Ring H, Tamir A. The spinal cord independence measure (SCIM): sensitivity to functional changes in subgroups of spinal cord lesion patients. Spinal Cord 2001; 39: 97-100.

Supplementary Information accompanies the paper on the Spinal Cord website (http://www.nature.com/sc) 